\title{
The Dynamics of Two Coupled Rigid Bodies
}

\author{
R. GROSSMAN,* P. S. KRISHNAPRASAD $\dagger$ AND JERROLD E. MARSDEN
}

\begin{abstract}
In this paper we derive a Poisson bracket on the phase space $80(3)^{*} \times \operatorname{so}(3)^{*} \times \mathrm{SO}(3)$ such that the dynamics of two three dimensional rigid bodies coupled by a ball and socket joint can be written as a Hamiltonian system.
\end{abstract}

\section{\$1. Introduction}

In this paper we introduce a Poisson bracket on the phase space

$$
\operatorname{so}(3)^{*} \times \operatorname{so}(3)^{*} \times \operatorname{SO}(3)
$$

where so $(3)^{*}$ is the dual of the Lie algebra of $S O(3)$, so that the dynamics of two rigid bodies coupled by a ball and socket joint can be written as the Hamilitonian system $\dot{H}=\{F, H\}$. This sets the stage so that the stability and asymptotics of the system can be studied using the energy Casimir method as in Holm, Marsden, Ratiu and Weinstein [1985] and Krishnaprasad [1985]; so that chaotic solutions can be found using the Melnikov method such as in Holmes and Marsden [1983]; so that bifurcations of the system can be described using the techniques in Golubitsky and Stewart [1986] and Lewis, Marsden and Ratiu [1986]; and so that control issues can be studied, as in Sanchez de Alvarez [1986].

The dynamies of planar coupled rigid bodies has been studied using similar ideas in Sreenath, Krishnaprasad and Marsden [1986].

Research supported in part by AFOSR-URI grant \# AFOSR-87-0073.

* Supported by an NSF postdoctoral fellowship held at the University of California, Berkeley.

tDepartment of Electrical Engineering and the Systems Research Center, University of

Maryland, College Park, Maryland, 20742. Partially supported by NSF grant

OIR-85-00108, and by the Minta Martin Fund for Aeronautical Research.

$¥$ Department of Mathematics, University of California, Berkeley, Berkeley, CA

94720. Research partially supported by DOE contract DE-AT03-85ER-12097. 


\section{\$2. Kinematics}

In this section we will derive the Lagrangian describing the free motion of two rigid bodies coupled by a ball and socket joint. At time 0 we assume that the two coupled rigid bodies are in a reference configuration denoted $\mathcal{B}$. Fix an inertial frame and let $Q$ denote a point in the reference configuration $\mathcal{B}$. Let $\mathcal{B}_{1}$ denote those points $Q \in \mathcal{B}$ which belong to body 1 and let $B_{2}$ denote those points which belong to body 2 .

The configuration at timet is determined by a smooth map

$$
\eta: B \longrightarrow \mathbf{R}^{3}, \quad Q \rightarrow q(Q, t) .
$$

We can also specify the configuration at time $t$ as follows. First we specify the position of the joint with respect to the inertial frame. Denote this as $w(t)$. Fix a frame centered at the joint and parallel to the inertial frame. With respect to this frame, the configuration of body 1 is determined as usual by three Euler angles. The Euler angles determine the orientation of a body fixed frame relative to the spatial frame centered at the joint. Alternatively these two frames are related by an element $A_{1}(t) \in S O(3)$. Similarly the configuration of body 2 is determined by an element $A_{2}(t) \in S O(3)$. We conclude that the configuration space is

$$
c=\mathrm{SO}(3) \times \mathrm{SO}(3) \times \mathrm{R}^{3}
$$

and that

$$
\begin{array}{ll}
q(Q, t)=A_{1}(t) Q+w(t), & \text { for } Q \in \mathcal{B}_{1} \\
q(Q, t)=A_{2}(t) Q+w(t), & \text { for } Q \in \mathcal{B}_{2} .
\end{array}
$$

We now proceed to compute the kinetic energy of the system. This requires that we keep track of the centers of mass of the two bodies and the center of mass of the system relative to the fixed inertial frame as well as the frame centered at the joint. Let $m_{1}$ and $m_{2}$ denote the masses of the two bodies and let $m$ denote the total mass. Let $S_{1}^{0}$ denote the center of mass of body 1 in the reference configuration relative to the inertial frame and let $S_{2}^{0}$ denote the center of mass for body 2. Let $r_{1}(t)$ denote the center of mass of body 1 at time $t$ relative to the inertial frame and let $r_{2}(t)$ denote the center of mass for body 2. Let $s_{1}(t)$ and $s_{2}(t)$ denote the center of mass of bodies 1 and 2 , respectively, measured with respect to the frame centered at the joint. Finally let $\alpha(t)$ denote the center of mass of the ensemble measured with respect to the inertial frame. Figures 1 and 2 show the relationships of these quantites. For example the following equations can be read off from the figures

$$
\begin{array}{ll}
s_{1}(t)=A_{1}(t) S_{1}^{0} & r_{1}(t)=w(t)+s_{1}(t) \\
s_{2}(t)=A_{2}(t) S_{2}^{0} & r_{2}(t)=w(t)+s_{2}(t)
\end{array}
$$
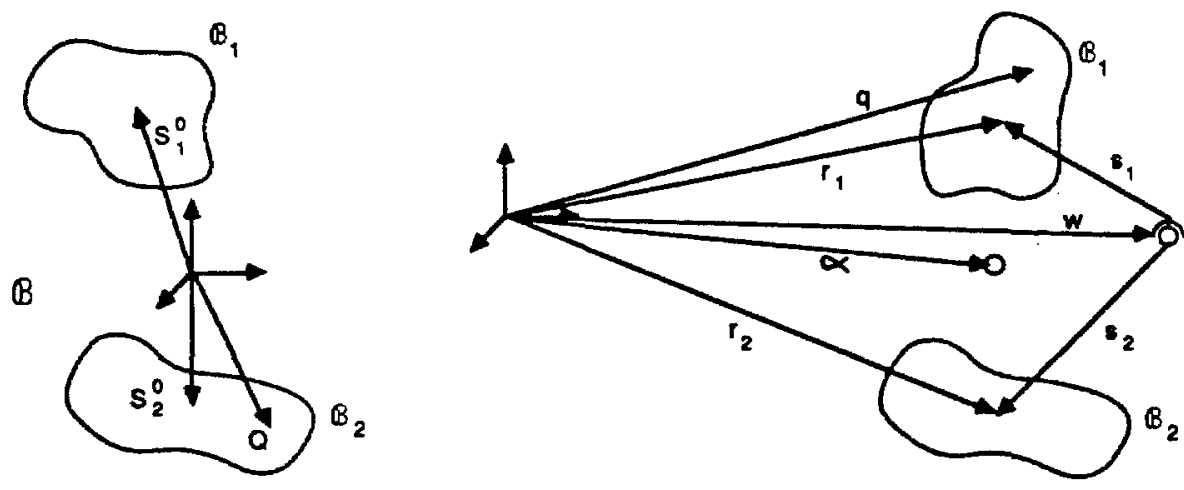
Given two vectors $v=\left(v^{1}, v^{2}, v^{3}\right)$ and $w=\left(w^{1}, w^{2}, w^{3}\right)$, denote their inner product relative to the standard Euclidean structure as

$$
(v, w)=\sum_{i=1}^{3} v^{i} w^{i},
$$

and the corresponding norm by $|v|$. Let $\mu(Q)$ denote the mass measure of the ensemble in the seference configuration. The kinetic energy $\mathrm{KE}$ of the configuration is

$$
\begin{aligned}
\mathrm{KE}= & \frac{1}{2} \int_{\mathcal{B}}|\dot{q}(t)| \mathrm{d} \mu(Q) \\
= & \frac{1}{2} \int_{\mathcal{B}_{1}}\left|\dot{A}_{1}(t) Q+w(t)\right|^{2} \mathrm{~d} \mu(Q)+(1 \leftrightarrow 2) \\
= & \frac{1}{2} \int_{\mathcal{B}_{1}}\left(\dot{A}_{1 k}^{i} Q^{k}+\dot{w}^{i}\right) \cdot\left(\dot{A}_{2 l}^{j} Q^{l}+\dot{w}^{j}\right) \mathrm{d} \mu(Q)+(1 \leftrightarrow 2) \\
= & \left\{\frac{1}{2} \int_{\mathcal{B}_{1}} Q^{k} Q^{l} \mathrm{~d} \mu(Q)\right\} \dot{A}_{1 k}^{i} \dot{A}_{1 !}^{j}+\left\{\frac{1}{2} \int_{\mathcal{B}_{1}} \mathrm{~d} \mu(Q)\right\} \dot{w}^{i} \dot{w}^{j} \\
& +\left\{\frac{1}{2} \int_{\mathcal{B}_{1}} Q^{k} \mathrm{~d} \mu(Q)\right\} \dot{A}_{1 k}^{i} \dot{w}^{j}+\left\{\frac{1}{2} \int_{\mathcal{B}_{1}} Q^{l} \mathrm{~d} \mu(Q)\right\} \dot{A}_{1 !}^{j} \dot{w}^{i}+(1 \leftrightarrow 2) .
\end{aligned}
$$

Let $I_{1}$ denote the coefficient of inertia matrix of body 1 , defined by

$$
\left(I_{1}\right)^{i j}=\int_{\mathcal{B}_{1}} Q^{i} Q^{j} \mathrm{~d} \mu(Q), \quad \text { for } i, j=1,2,3 .
$$

The coefficient of inertia $\tilde{I}_{2}$ of body 2 is defined similarly. Using these definitions and the defintion of the center of mass, we can rewrite the expressions above as

$$
\begin{aligned}
\mathrm{KE} & =\frac{1}{2} \operatorname{tr}\left(\dot{A}_{1} I_{1} \dot{A}_{1}^{t}\right)+\frac{m_{1}}{2}\left(\dot{w}^{\prime}, \dot{w}\right)+m_{1}\left(\dot{A}_{1} S_{1}^{0}, \dot{w}\right)+(1 \leftrightarrow 2) \\
& =\frac{1}{2} \operatorname{tr}\left(\dot{A}_{1} I_{1} \dot{A}_{1}^{t}\right)+m_{1}\left(\dot{A}_{1} S_{1}^{0}, \dot{w}\right)+(1 \leftrightarrow 2)+\frac{m}{2}(\dot{w}, \dot{w})
\end{aligned}
$$

The Lagrangian is simply the total kinetic energy. To summarize, the velocity phase space for our system is $T \mathrm{SO}(3) \times T \mathrm{SO}(3) \times T \mathbf{R}^{3}$ and the Lagrangian is given by (2.5).

\section{\$3. Reduction by the Euclidean Group}

Consider the following action of an element $g$ of the Euclidean group $E(3)$

$$
g=\left(\begin{array}{ll}
B & b \\
0 & 1
\end{array}\right)
$$

where $A \in \mathrm{SO}(3)$ and $b \in \mathbf{R}^{3}$, on a point $\left(A_{1}, A_{2}, w\right)$ in the configuration space $\mathcal{C}$ :

$$
g \cdot\left(A_{1}, A_{2}, w\right)=\left(B A_{1}, B A_{2}, B w+b\right) .
$$

It is easy to check that the Lagrangian (2.5) is invariant under this action. Since the Lagrangian is invariant under $E(3)$, so is the Hamiltonian. The purpose of this section is to perform the reduction by this group. This will be done in two steps. First we will reduce by $\mathbf{R}^{3}$; this accounts for conservation of total linear momentum. Then we will reduce by $\mathrm{SO}(3)$; this accounts for conservation of total angular momentum.

We begin by rewriting the Lagragian in terms of the linear momentum p. Using (2.2), we can write the total linear momentum as

$$
\begin{aligned}
p & =m \dot{\alpha}=m_{1} \dot{r_{1}}+m_{2} \dot{r_{2}} \\
& =m \dot{w}+m_{1} \dot{A_{1}} S_{1}^{0}+m_{2} \dot{A_{2}} S_{2}^{0} .
\end{aligned}
$$


It is convenient to introduce the expression

$$
\rho=\frac{m_{1}}{m} \dot{A}_{1} S_{1}^{0}+\frac{m_{2}}{m} \dot{A}_{2} S_{2}^{0} .
$$

and write

$$
\begin{aligned}
w & =\alpha-\frac{m_{1}}{m} A_{1} S_{1}^{0}-\frac{m_{2}}{m} A_{2} S_{2}^{0} \\
\dot{w} & =\dot{\alpha}-\rho
\end{aligned}
$$

Substituting (3.3a and $b$ ) into (2.5) and simplifying gives the following form for the Lagrangian

$$
L=\frac{1}{2} \operatorname{tr}\left(\dot{A}_{1} I_{1} \dot{A}_{1}^{t}\right)+\operatorname{tr}\left(\dot{A}_{2} I_{2} \dot{A}_{2}^{q}\right)+\frac{1}{2 m}(p, p)-\frac{m}{2}(\rho, \rho) .
$$

The Legendre transformation $F L$ induces a symplectic structure on

$$
T C=T S O(3) \times T S O(3) \times T R^{3}
$$

and the tangent of the action of $\mathbf{R}^{3}$ on $\mathcal{C}$ (see (3.1)) is symplectic. To verify this statement and the ones that follow, Abraham and Marsden [1978], chapters 3 and 4. The momentum map for this action is given by

$$
\begin{gathered}
J: T S O(3) \times T \mathrm{SO}(3) \times T \mathrm{R}^{3} \longrightarrow \mathrm{R}^{3^{*}} \\
\left(A_{1}, \dot{A}_{1}, A_{2}, \dot{A}_{2}, w, \dot{w}\right) \mapsto p .
\end{gathered}
$$

The corresponding reduced space at $p$ is:

$$
\left(J^{-1}(p)\right) / \mathrm{R}^{3}=T S O(3) \times T S O(3) .
$$

From (3.4) we see that the Lagrangian on the reduced space is simply

$$
L=\frac{1}{2} \operatorname{tr}\left(\dot{A}_{1} I_{1} \dot{A}_{1}^{t}\right)+\frac{1}{2} \operatorname{tr}\left(\dot{A}_{2} I_{2} \dot{A}_{2}^{t}\right)-\frac{1}{2 m}\left|m_{1} \dot{A}_{1} S_{1}^{0}+m_{2} \dot{A}_{2} S_{2}^{0}\right|^{2}+\frac{|p|^{2}}{2 m}
$$

Since $p$ is constant, we can drop the last term. This completes the first stage of the reduction.

We now perform the reduction corresp onding to conservation of total angular momentum. This time we use Poisson reduction; see Krishnaprasad and Marsden [1986] for a summary of Poisson reduction. Consider the map

$$
\begin{aligned}
& \lambda: T^{*}(\mathrm{SO}(3) \times \mathrm{SO}(3)) \longrightarrow \mathrm{so}(3)^{*} \times \mathrm{so}(3)^{*} \times \mathrm{SO}(3)
\end{aligned}
$$

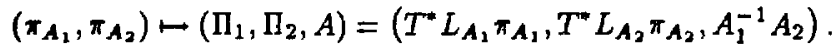

We will define a Poisson bracket on the target space so that the map $\lambda$ becomes a Poisson map with respect to this bracket and the canonical bracket on the cotangent bundle $T^{*}(\mathrm{SO}(3) \times \mathrm{SO}(3))$.

Introduce the body angular velocities of each of the bodies

$$
\begin{aligned}
& \hat{\Omega}_{1}=A_{1}^{-1} \dot{A}_{1} \\
& \hat{\Omega}_{2}=A_{2}^{-1} \dot{A}_{2}
\end{aligned}
$$

where $\hat{\Omega}$ is the linear map $v \mapsto \Omega \times v$ on $\mathbf{R}^{3}$. We will also need the moment of inertia $J_{1}$ of body 1 given by

$$
\begin{aligned}
\left(J_{1}\right)^{i j} & =-\int_{\mathcal{B}_{1}} Q^{i} Q^{j} \mathrm{~d} \mu(Q), \quad \text { if } i \neq j \\
& =\int_{\mathcal{B}_{1}}\left(|Q|^{2}-\left(Q^{i}\right)^{2}\right) \mathrm{d} \mu(Q), \quad \text { if } i=j .
\end{aligned}
$$

The moment of inertia $J_{2}$ of body 2 is defined in a similar way. 
In order to derive an explicit exprssion for the angular momenta of the system, it is helpful to write the total kinetic energy as a quadratic form. After a certain amount of algebra, the total kinetic energy (3.5), can be written

where

$$
\left(\Omega_{1}^{\ddagger}, \Omega_{2}^{\ddagger}\right)\left(\begin{array}{cc}
\tilde{J}_{1} & \epsilon \Lambda \\
\epsilon \Lambda^{*} & \tilde{J}_{2}
\end{array}\right)\left(\Omega_{1}, \Omega_{2}\right)
$$

Let $\mathbf{J}$ denote the matrix

$$
\begin{aligned}
\tilde{J}_{1} & =J_{1}+\frac{m_{1}^{2}}{m}\left(\left|S_{1}^{0}\right|^{2} \cdot 1-S_{1}^{0} \otimes S_{1}^{0}\right) \\
\tilde{J}_{2} & =J_{2}+\frac{m_{2}^{2}}{m}\left(\left|S_{2}^{0}\right|^{2} \cdot 1-S_{2}^{0} \otimes S_{2}^{0}\right) \\
\Lambda & =\left(\hat{S}_{1}^{0}\right)^{\dagger} A\left(\hat{S}_{2}^{0}\right) \\
\epsilon & =\frac{m_{1} m_{2}}{m}
\end{aligned}
$$

From the Legendre transformation applied to Lagrangians quadratic in the velocities, the angular momenta are found to be

$$
\left(\begin{array}{l}
\mathbf{M}_{1} \\
\Pi_{2}
\end{array}\right)=\mathbf{J}\left(\begin{array}{l}
\Omega_{1} \\
\Omega_{2}
\end{array}\right)
$$

The inertial orientation matrices $A_{1}, A_{2}$ determine the relative orientation matrix

$$
A=A_{1}^{-1} A_{2} \text {. }
$$

Using the definitions (3.7) - (3.10), we can rewrite the Lagragian (3.5) as

$$
L=\frac{1}{2} \Pi \cdot \mathbf{J}^{-1} \Pi
$$

and conclude, using the fact that the Lagragian is quadratic in the momenta, that the Hamiltonian $H\left(\Pi_{1}, \Pi_{2}, A\right)$ is also given by $(3.11)$.

We next derive the Poisson bracket on $\mathrm{so}(3)^{*} \times \operatorname{so}(3)^{*} \times \mathrm{SO}(3)$. Given a function $F$ on $\operatorname{so}(3)^{*} \times$ so $(3)^{*} \times \mathrm{SO}(3)$, define a function $F_{\lambda}$ on $T^{*}(\mathrm{SO}(3) \times \mathrm{SO}(3))$ by

$$
F_{\lambda}=F \circ \lambda \text {. }
$$

The canonical bracket on $T^{*}(\mathrm{SO}(3) \times \mathrm{SO}(3))$ is

$$
\begin{aligned}
\left\{F_{\lambda}, G_{\lambda}\right\}= & D_{A_{1}} F_{\lambda} \cdot \frac{\delta H_{2}}{\delta \pi_{1}}-D_{A_{1}} H_{\lambda} \cdot \frac{\delta F_{2}}{\delta \pi_{1}} \\
& +D_{A_{2}} F_{\lambda} \cdot \frac{\delta H_{\lambda}}{\delta \pi_{2}}-D_{A_{2}} H_{\lambda} \cdot \frac{\delta F_{2}}{\delta \pi_{2}} .
\end{aligned}
$$

Using the chain rule, we can introduce a bracket on $\mathrm{so}(3)^{*} \times \mathrm{so}(3)^{*} \times \mathrm{SO}(3)$, so that $\lambda$ becomes a Poisson map. This is straghtforward but tedious. To organize the computation, it is helpful to note the following facts.

Fact 1. If $\pi_{A} \in T_{A}^{*} S O(3)$, then

$$
\text { II : }=T_{e}^{*} L_{A} \cdot \pi_{A}=A^{t} \pi_{A} \in \operatorname{so}(3)^{*}
$$

Fact 2. Let $H_{\lambda}\left(A_{1}, \pi_{1}, A_{2}, \pi_{2}\right)$ be a function on $T^{*}(S O(3) \times S O(3))$ and let $\frac{\delta H_{\lambda}}{\delta \pi_{2}}$ denote the functional derivative of $H_{\lambda}$ with respect to $\pi_{1}$. Then

$$
\frac{\delta H_{2}}{\delta \pi_{1}}=A \frac{\delta H}{\delta \Pi_{1}} \text {. }
$$

Fact 3. Let $F_{\lambda}\left(A_{1}, \pi_{1}, A_{2}, \pi_{2}\right)$ be a function on $T^{*}(S O(3) \times S O(3))$, where we have, by abuse of notation, written an element in the cotangent space at $A_{1}$ as $\left(A_{1}, \pi_{1}\right)$. Then

$$
D_{A_{1}} F_{\lambda}\left(A_{1}, \pi_{1}, A_{2}, \pi_{2}\right)\left(\delta A_{1}\right)=\left\langle\frac{\delta F}{\delta \pi_{2}},\left(\delta A_{2}\right)^{t} \pi_{2}\right\rangle+\left\langle\frac{\delta F}{\delta A}, A_{1}^{-1}\left(\delta A_{2}\right)\right\rangle
$$

It is now straightforward to combine these facts to see that the canonical bracket (3.13) may be written in terms of the fuctions $F$ and $G$ using the chain rule as

$$
\begin{aligned}
\{F, H\}\left(\Pi_{1}, \Pi_{2}, A\right)=- & \left\langle\Pi_{1},\left[\frac{\delta F}{\delta \Pi_{1}}, \frac{\delta H}{\delta \Pi_{1}}\right]\right\rangle-\left\langle\Pi_{2},\left[\frac{\delta F}{\delta \Pi_{2}}, \frac{\delta H}{\delta \Pi_{2}}\right]\right\rangle \\
& -\left\langle\frac{\delta F}{\delta A}, \frac{\delta H}{\delta \Pi_{1}} A-A \frac{\delta H}{\delta \Pi_{2}}\right\rangle+\left\langle\frac{\delta H}{\delta A}, \frac{\delta F}{\delta \Pi_{1}} A-A \frac{\delta E}{\delta H_{2}}\right\rangle .
\end{aligned}
$$

To summarize, we have 
Theorem 1. (i) With the canonical bracket on $T^{*}(S O(3) \times S O(3))$ and the bracket (3.15) on $\mathrm{so}(3)^{*} \times \mathrm{so}(3)^{*} \times S O(3)$, the map

$$
\lambda: T^{*}(S O(3) \times S O(3)) \longrightarrow s o(3)^{*} \times \mathrm{so}(3)^{*} \times S O(3)
$$

given by (3.6) is a Poisson map. (ii) The dynamics on the reduced space is given by

$$
\dot{F}=\{F, H\}
$$

\section{§4. Further Remarks}

1. We begin with a brief discussion of the Casimirs. Consider the momentum map

$$
\begin{aligned}
& J: T^{*}(\mathrm{SO}(3) \times \mathrm{SO}(3)) \longrightarrow \operatorname{so}(3)^{*} \\
& \left(\pi_{A_{1}}, \pi_{A_{2}}\right) \mapsto\left(T^{*} R_{A_{1}} \pi_{A_{1}}+T^{*} R_{A_{2} \pi_{A_{2}}}\right) .
\end{aligned}
$$

The composition of this with the Casimir

$$
\begin{aligned}
C: \mathrm{so}(3)^{*} & \longrightarrow \mathbf{R} \\
\mathbf{I} & \mapsto|\Pi|^{2}
\end{aligned}
$$

produces a collective Hamiltonian on $T^{*}(\mathrm{SO}(3) \times \mathrm{SO}(3))$ whose Hamiltonian vector field is tangent to the $G$-orbits (see Guillemin and Sternberg [1980] or Holmes and Marsden [1983]) and therefore induces a Casimir $\tilde{C}$ on the space so $(3)^{*} \times s o(3)^{*} \times S O(3)$ via the Poisson map (3.6).

Tracing through the diagram shows that $\tilde{C}=\left|\Pi_{1}+A \Pi_{2}\right|^{2}$; and, hence, any function of the form $\Phi\left(\left|\Pi_{1}+A \Pi_{2}\right|^{2}\right)$ is a Casimir for the bracket (3.15).

2. The symplectic leaves in the nine dimensional space so(3) $\times 8 \mathrm{so}(3)^{*} \times \mathrm{SO}(3)$ appear to be eight dimensional (level sets of the function $\left|\Pi_{1}+A \Pi_{2}\right|^{2}$ ) and in the case of $J=0$, (given by (4.1), the six dimensional space $T^{*} S O(3)$; and, finally, if $\Pi_{1}=0, \Pi_{2}=0$, a two dimensional space $S^{2}$ of trival equilbria. We expect to explore the geometry of these leaves and the other topics listed in the introduction in a future publication.

\section{References} Wesley.

Abraham, R. and Marsden, J. [1978] Foundations of Mechanics, second edition, Addison-

Guillemin, V. and Sternberg, S. [1980], The Moment Map and Collective Motion, Ann. of Phys., vol. 127, pp. 220-253.

Golubitsky, M. and Stewart [1986], I., Generic Bifurcation of Hamiltonian Systems with Symmetry to appear in Physica $D$.

Holm, D., Marsden, J., Ratiu, T., and Weinstein, A. [1985], Nonlinear Stability of Fluid and Plasma Equilbria, Physics Reports, vol. 123, nos. 1-2, pp. 1-116.

Holmes, P. and Marsden [1983], J., Horseshoes and Arnold Diffusion for Hamiltonian Systems on Lie Groups, Indiana University Mathematics Journal, vol. 32, no. 2, pp. 273-309.

Krishnaprasad, P. [1985], Lie-Poisson Structures, Dual-Spin Spacecraft, and Asymptotic Stability, Nonlinear Analysis, Theory, Methods and Appl., vol. 9, pp. 1011-1035.

Krishnaprasad, P. and Marsden, J. [1987], Hamiltonian Structures and Stability for Rigid Bodies with Flexible Attachments, Arch. Rat. Mech. Anal., vol. 98, no. 1, pp. 71-93.

Lewis, D., Marsden, J. and Ratiu, T. [1986], Stability and Bifurcation of Rotating Planar Liquid Drop, Tech. Rep. PAM - 330, Center For Pure and Applied Mathematics, University of California, Berkeley.

Sanchez de Alvarez, G. [1986], Geometric Methods of Classical Mechanics Applied to Control Theory, Ph. D. thesis, University of California, Berkeley.

Sreenath, N., Krishnaprasad, P. and Marsden, J. [1986], The Dynamics of Coupled Planar Rigid Bodies, Systems Research Center Technical Report TR - 86 - 56, University of Maryland, College Park. 